\title{
Localization in a disordered multi-mode waveguide with absorption or amplification
}

\author{
T.Sh. Misirpashaev ${ }^{\mathrm{a}, \mathrm{b}, *}$, J.C.J. Paasschens ${ }^{\mathrm{a}, \mathrm{c}}$, C.W.J. Beenakker ${ }^{\mathrm{a}}$ \\ "Instutuut-Lonentz, Unveisty of Leiden, PO Bo 9506, 2300 RA Leiden, The Netheilunds \\ ${ }^{b}$ Landau Institure for Theoretual Physics, 2 Kosygm Street, Moscon 117334, Russia \\ ${ }^{c}$ Phllps Research Laboratones, 5656 AA Endhoven The Netherlands
}

Rccelved 9 September 1996

\begin{abstract}
An analytical and numerical study of tiansmission of radiation through a multi-mode wavegurde containing a 1andom medium with a complex dielectric constant $\varepsilon=\varepsilon^{\prime}+t \varepsilon^{\prime \prime}$ is presented Depending on the sign of $\varepsilon^{\prime \prime}$, the medium is absorbing or amplifyng. The transmitted intensity decays exponentially $\propto \exp (-L / \zeta)$ as the waveguide length $L \rightarrow \infty$, regardless of the sign of $\varepsilon^{\prime \prime}$ The localization length $\xi$ is computed as a function of the mean frec path $l$, the absorption or amplification length $|\sigma|^{-1}$, and the number of modes in the wavegude $N$. The method used is an extension of the Fokker-Planck approach of Dorokhov, Mcllo, Pereyra and Kumar to nonunitary scattering matrices. Asymptotically exact results aie obtaincd for $N \gg 1$ and $|\sigma| \gg 1 / N^{2} l$. An approximate interpolation formula for all $\sigma$ agiees reasonably well with numerical sumulations
\end{abstract}

PACS. 78.45.+h, 42.25.Bs; 72.15.Rn; $7820 \mathrm{Cl}$

\section{Introduction}

Localization of waves in one-dimensional random media has been studied extensively, both for optical and for electronic systems [1,2]. An analytical solution for the case of weak disorder (mean free path $l$ much greater than the wavelength $\lambda$ ) was obtained as early as 1959 by Gertsenshtein and Vasil'ev [3]. The transmittance $T$ (being the ratio of transmitted and incident intensity) has a log-normal distribution for large lengths $L$ of the system, with a mean $\langle\ln T\rangle=-L / \xi$ characterized by a localization length $\xi$ equal to the mean free path.

*Corresponding author Fax +31715275404, c-mail. missı@louentz leidenuniv.n! 
This early work was concerned with the propagation of classical waves, and hence included also the effect of absorption In the presence of absorption the transmittance decays faster, according to $[4,5]\langle\ln T\rangle=\left(\sigma-l^{-1}\right) L$, where $|\sigma|$ is the inverse absorption length $(\sigma<0)$ Absorption is the result of a positive 1maginary pait $\varepsilon^{\prime \prime}$ of the (1elative) dielecti1c constant $\imath=\imath^{\prime}+\iota^{\prime \prime}$ For a homogeneous $\varepsilon^{\prime \prime}$ one has

$$
\sigma=-2 k \operatorname{Im} \sqrt{1+l \varepsilon^{\prime \prime}} \approx-k \varepsilon^{\prime \prime} \text { if }\left|\varepsilon^{\prime \prime}\right| \ll 1,
$$

where $k$ is the wavenumber A negative $\varepsilon^{\prime \prime}$ corresponds to amplification by stimulated emission of radiation, with inverse amplification length $\sigma>0$ Piopagation of waves through amplifying one-dimensional random media has been studied in [6-11] In the limit $L \rightarrow \infty$ amplification also leads to a faster decay of the transmittance, accoiding to $\langle\ln T\rangle=\left(-|\sigma|-l^{-1}\right) L[8,9]$

A natural extension of these studies is to waveguides which contain more than a single propagating mode Localization in such "quasi-one-dimensional" systems has been studied on the basis of a scaling theory [12], a supersymmetric field theory [13], or a Fokker-Planck equation $[14,15]$ It is found that the localization length for $N$ modes 1 s enhanced by a factor of o1der $N$ relative to the single-mode case These investigations were concerned with quantum mechanical, rathe1 than classical waves, and therefore did not include absorption It $1 \mathrm{~s}$ the puipose of the present paper to extend the Fokker Planck approach of Dorokhov, Mello, Pereyı a and Kumar [14,15] (DMPK) to include the effects on the transmittance of a non-zero 1maginary part of the dielectric constant

According to the general duality ielation [9], the localization length is an even function of $\sigma$ for any $N$,

$$
\xi(\sigma)=\xi(-\sigma)
$$

It follows that both absorption and amplification lead to a faster decay of the transmittance for large $L$ For $N \gg 1$ we find that, to a good approximation,

$$
\frac{1}{\xi}=\frac{2}{(N+1) l}+\left(\sigma^{2}+2|\sigma| / l\right)^{1 / 2}
$$

This result becomes exact in the two limits $|\sigma| \gg 1 / N^{2} l$ and $|\sigma| \ll 1 / N^{2} l$ We compare with numerical solutions of the Helmholtz equation, and find reasonably good agreement over the whole range of $\sigma$

The outline of the paper is as follows In Section 2 we formulate the scattering problem and summarize the duality relation of [9] In Section 3 we delive a Fokker Planck equation for the transmission and reflection eigenvalues $\mathscr{T}_{n}, \mathscr{R}_{n}, n=1,2, \quad, N$ These are eigenvalues of the matrix products $t t^{\dagger}$ and $r r^{\dagger}$, respectively, where $t$ and $r$ are the transmission and reflection matrices of the waveguide For $\sigma=0$ the FokkerPlanck equation is the DMPK equation [14,15] A reduced Fokke1 Planck equation, containing only the $\mathscr{R}_{n}$ 's, was previously obtained and studied 11 [16,17] To obtain the localization length one needs to include also the $\mathscr{T}_{n}$ 's, which are no longer related to the $\mathscr{R}_{n}$ 's when $\sigma \neq 0$ We find that a closed Fokker-Planck equation containing 
$\mathscr{R}_{n}$ 's and $\mathscr{T}_{n}$ 's exists only for $N=1$ If $N>1$ there appears an additional set of "slow variables," consisting of eigenvectors of $t r^{\dagger}$ in a basis where $t t^{\dagger}$ is diagonal (These new variables do not appear when $\sigma=0$, because then $r^{\dagger}$ and $t t^{\dagger}$ commute ) Because of these additional relevant vaniables we have not been able to make as much pıogiess in the solution of the Fokkel-Planck equation for $\sigma \neq 0$ as one can for $\sigma=0$ [18] In Section 4 we show that a closed evolution equation for $\langle\ln T\rangle$ can be obtained if $|\sigma| \gg 1 / N^{2} l$, which leads to the second term in Eq (13) (This term could also have been obtained from the incoherent radiatıve transfer theory for $\sigma<0$, but not for $\sigma>0)$ To contrast the multi-mode and single-mode cases, we also butefly discuss in Section 4 the derivation of the localization length for $N=1$ (Our $N=1$ results were given without deivation in [9] ) Finally, in Section 5 we compaie the analytical results for the multi-mode case with numeical simulations

\section{Formulation of the scattering problem}

We consider a 1andom medium of length $L$ with a spatially fluctuating dielectric constant $\varepsilon=\varepsilon^{\prime}+\imath \varepsilon^{\prime \prime}$, embedded in an $N$-mode waveguide with $\varepsilon=1$ The scatteing matux $S$ is a $2 N \times 2 N$ matıx ielating incoming and outgoing modes at some fiequency $\omega$ It has the block stiucture

$$
S=\left(\begin{array}{cc}
r^{\prime} & t^{\prime} \\
t & r
\end{array}\right),
$$

where $t, t^{\prime}$ are the transmission matıces and $r, r^{\prime}$ the reflection matrices We introduce the sets of transmission and ieflection eigenvalues $\left\{\mathscr{T}_{n}\right\},\left\{\mathscr{T}_{n}^{\prime}\right\},\left\{\mathscr{R}_{n}\right\},\left\{\mathscr{R}_{n}^{\prime}\right\}$, being the ergenvalues of $t t^{\dagger}, t^{\prime} t^{\prime}, r r^{\dagger}, r^{\prime}{ }^{\prime \dagger}$, respectively Total transmittances and reflectances are defined by

$$
\begin{array}{ll}
T=N^{-1} \operatorname{Tr} t t^{\prime}, & R=N^{-1} \operatorname{Tr} r r^{\prime}, \\
T^{\prime}=N^{-1} \operatorname{Tr} t^{\prime} t^{\prime}, & R^{\prime}=N^{-1} \operatorname{Tr} t^{\prime} t^{\prime \dagger}
\end{array}
$$

Here $T$ and $R^{\prime}$ are the transmitted and ieflected intensity divided by the incident intensity from the left Similaily, $T^{\prime}$ and $R$ coriespond to incident intensity fiom the right By taking the trace in Eq (22) we are assuming diffuse illumination, $1 \mathrm{e}$ that the incident intensity is equally distributed over the $N$ modes Two systems which differ only 1 the sign of $\varepsilon^{\prime \prime}(\boldsymbol{r})$ are called dual Scattering matices of dual systems are related by [9]

$$
S\left(\varepsilon^{\prime \prime}\right) S^{\prime}\left(-\varepsilon^{\prime \prime}\right)=1
$$

This duality ielation takes the place of the unitaity constraint when $\varepsilon^{\prime \prime} \neq 0$

An optical system usually possesses time-1eversal symmetiy, as a iesult of which $S\left(\varepsilon^{\prime \prime}\right) S^{*}\left(-\varepsilon^{\prime \prime}\right)=1$ Combining this telation with Eq (23), we find that $S=S^{\mathrm{T}}$ is a symmetric matrix Hence $\mathscr{T}_{n}=\mathscr{T}_{n}^{\prime}$ and $T=T^{\prime}$ (The reflectances $R$ and $R^{\prime}$ may differ ) The case of broken time-1eversal symmetty might also be physically relevant [19], and 
is included here for completeness In the absence of time-1eversal symmetry $S$ is an arbitrary complex matrix

The duality relation (2 3) has consequences for the teflection and tiansmission eigenvalues of two dual systems [9] If $N=1$ the ielation

$$
T\left(\varepsilon^{\prime \prime}\right) / R\left(\varepsilon^{\prime \prime}\right)=T^{\prime}\left(-r^{\prime \prime}\right) / R^{\prime}\left(-\varepsilon^{\prime \prime}\right)
$$

holds for all $L$ If $N \geqslant 1$ we have two relations for $L \rightarrow \infty$,

$$
\begin{aligned}
& \lim _{L \rightarrow \infty} \mathscr{R}_{n}\left(\varepsilon^{\prime \prime}\right)=\lim _{L \rightarrow \infty} \mathscr{R}_{n}^{1}\left(-\varepsilon^{\prime \prime}\right), \\
& \lim _{L \rightarrow \infty} L^{-1} \ln \mathscr{T}_{n}\left(\varepsilon^{\prime \prime}\right)=\lim _{L \rightarrow \infty} L^{-1} \ln \mathscr{T}_{n}\left(-\varepsilon^{\prime \prime}\right)
\end{aligned}
$$

The transmittance $T=N^{1} \sum_{n} \mathscr{T}_{n} 1$ s dominated by the largest transmission eigenvalue, hence

$$
\lim _{L \rightarrow \infty} L^{-1} \ln T\left(\varepsilon^{\prime \prime}\right)=\lim _{l \rightarrow \infty} L^{-1} \ln T\left(-\varepsilon^{\prime \prime}\right)
$$

In other words, two dual systems have the same localization length, as stated in $\mathrm{Eq}\left(\begin{array}{ll}1 & 2\end{array}\right)$

\section{Fokker-Planck equation}

We derive a Fokker-Planck equation for the evolution of the distribution of scatteing matrices with increasing length $L$ of the waveguide In the absence of gain or loss $(\sigma=$ 0 ), the evolution equation is known as the Dorokhov-Mello-Pcieyra Kumar (DMPK) equation [14,15] Original derivations of this equation relied on the unitarity of the scattering matrix, making use of the invailant medsule on the unitary gioup and the polar decomposition of a unitary matıx These derivations cannot readily be generalized to the case $\sigma \neq 0$, in particular because the scatteing matıx no longer admits a polai decomposition (This means that the mat11x pioducts $n^{\dagger}$ and $t t^{\dagger}$ do not commute ) The alternative derivation of the DMPK equation [18] does not use the polai decomposition and is suitable for our purpose

Without loss of generality we can wite the transmission and ieflection submatuces of the scatteing matrix as follows,

$$
S=\left(\begin{array}{cc}
r^{\prime} & t^{\prime} \\
t & l
\end{array}\right)=\left(\begin{array}{cc}
U \sqrt{\mathbf{R}^{\prime}} W & U^{\prime} \sqrt{\mathbf{T}^{\prime}} Z \\
V \sqrt{\mathbf{T}} W^{\prime} & -V^{\prime} \sqrt{\mathbf{R}} Z^{\prime}
\end{array}\right)
$$

Here $U, U^{\prime}, V, V^{\prime}, W, W^{\prime}, Z, Z^{\prime}$ are $N \times N$ unitary matıces, while $\mathbf{R}, \mathbf{R}^{\prime}, \mathbf{T}, \mathbf{T}^{\prime}$ are didgonal matrices whose elements are the ieflection and tiansmission eigenvalues $\left\{\mathscr{R}_{n}\right\},\left\{\mathscr{R}_{n}^{\prime}\right\}$, $\left\{\mathscr{T}_{n}\right\},\left\{\mathscr{T}_{n}^{\prime}\right\}$ For $\sigma=0$, the unitarity constiaint $S S^{\top}=1$ implies $U=U^{\prime}, V=V^{\prime}$, $W=W^{\prime}, Z=Z^{\prime}$, and $\mathbf{R}=\mathbf{R}^{\prime}=1-\mathbf{T}=1-\mathbf{T}^{\prime}$ Eq (31) then constitutes the polar decomposition of the scattering matix In this case one can derive a Fokker Planck equation for the evolution of only tiansmission or only ieflection eigenvalues if $\sigma \neq 0$, 
the Fokker Planck equation contains both the transmission and reflection eigenvalues, as well as elements of the mat11x $Q=V^{\dagger} V^{\prime}$ elating eigenvectors of $t t^{\dagger}$ and $n_{1}^{\dagger}$ The only constiaint on the scatteing mat11x if $\sigma \neq 0$ 1s imposed by time-1eve1sal symmetry, which requires $S=S^{\mathrm{T}}$, hence $W=U^{\mathrm{T}} \quad Z=V^{\mathrm{T}}, W^{\prime}=U^{\prime \mathrm{T}}, Z^{\prime}=V^{\prime \mathrm{T}}, \mathbf{T}=\mathbf{T}^{\prime}$

The Fokke1-Planck equation descibes the evolution of slow variables after the elimination of fast variables In oul problem fast variables vary on the scale of the wavelength $\Lambda$, while slow variables vary on the scale of the mean fiee path $l$ or the amplification length $|\sigma|^{-1}$ We assume that both $l$ and $|\sigma|^{-1}$ are much gieate than $\lambda$ (This requiles $\left|\varepsilon^{\prime \prime}\right| \ll 1$ ) The slow valiables mclude $\left\{\mathscr{R}_{n}\right\},\left\{\mathscr{T}_{n}\right\}$ and elements of $Q=V^{\dagger} V^{\prime}$ We denote this set of slow valuables collectively by $\left\{\Phi_{n}\right\}$ Each $\Phi_{1}$ is

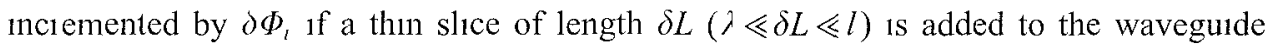
of length $L$ The inciements are of orde1 $(\delta L / l)^{1 / 2}$ and can be calculated perturbatively We specify an appropirate statistical ensemble for the scattering matix $\delta S$ of the thin slice and compute moments of $\delta \Phi_{l}$. The first two moments are of order $\partial L / l$,

$$
\begin{aligned}
& \left\langle\delta \Phi_{l}\right\rangle=a_{l} \delta L / l+\mathcal{O}(\delta L / l)^{3 / 2}, \\
& \left\langle\delta \Phi_{l} \partial \Phi_{l}\right\rangle=a_{l l} \partial L / l+\mathcal{O}(\partial L / l)^{3 / 2}
\end{aligned}
$$

Higher moments have no term of order $\delta L / l$ According to the general theory of Biownian motion [20], the Fokkeı-Planck equation for the joint probability distribution $P\left(\left\{\Phi_{n}\right\}, L\right)$ leads

$$
l \frac{\partial P}{\partial L}=-\sum_{l} \frac{\partial}{\partial \Phi_{l}} a_{l} P+\frac{1}{2} \sum_{l} \frac{\partial^{2}}{\partial \Phi_{l} \partial \Phi_{j}} a_{l} P
$$

The ave1agc $\langle>$ in Eq (32) is defined by the statistics of $\delta S$ We specify this statıstics using simplifying features of the waveguide geometiy (length $\gg$ width), which justify the equivalent channel or 1sotiopy approximation [15,21] We assume that amplification or absoiption in the thin slice is independent of the scatteing channel This entals the relation

$$
\delta S \delta S^{\dagger}=1+\bar{\sigma} \delta L
$$

where $\bar{\sigma}$ is a modal and spatial average of the inverse amplification length $\sigma$ If $\varepsilon^{\prime \prime}$ is spatially constant, one has

$$
\bar{\sigma}=-\frac{2 k}{N} \sum_{n 1}^{N} \operatorname{Im}\left(1-\omega_{n}^{2} / \omega^{2}+\imath \varepsilon^{\prime \prime}\right)^{1 / 2},
$$

where $\omega_{n}$ is the cutoff fiequency of mode $n$ For $N \rightarrow \infty$, the sum over modes can be replaced by an integial The 1esult depends on the dimensionality of the waveguide,

$$
\begin{array}{ll}
\bar{\sigma}=-2 k \varepsilon^{\prime \prime} & \text { for a 3D waveguide }, \\
\bar{\sigma}=-(\pi / 2) k \varepsilon^{\prime \prime} & \text { for a } 2 \mathrm{D} \text { wavegurde },
\end{array}
$$

where we have used that $\left|\varepsilon^{\prime \prime}\right| \ll 1$ 
Eq (3 4) ensules the existence of a polar decomposition for $\delta S$,

$$
\delta S=\left(\begin{array}{cc}
U_{0} \sqrt{\delta \mathbf{R}} W_{0} & U_{0} \sqrt{\delta \mathbf{T}} Z_{0} \\
V_{0} \sqrt{\delta \mathbf{T}} W_{0} & -V_{0} \sqrt{\delta \mathbf{R}} Z_{0}
\end{array}\right),
$$

with $\delta \mathbf{T}+\delta \mathbf{R}=1+\bar{\sigma} \delta L$ Note that a polar decomposition for $\delta S$ does not imply a pola1 decomposition for $S$, because the special block structure of $\mathrm{Eq}$ (3 7) is lost upon composition of scattering matrices We make the 1sotropy assumption that the matrices $U_{0}, V_{0}, W_{0}, Z_{0}$ are uniformly distributed in the unitary g1oup In the presence of timereversal symmetry one has $W_{0}=U_{0}^{\mathrm{T}}$ and $Z_{0}=V_{0}^{\mathrm{T}}$ In the absence of time-reversal symmetry all four unitary matrices are independent The didgonal matıces $\delta \mathbf{R}$ and $\delta \mathbf{T}$ may have arbitrary distributions We specify the first moments,

$$
\langle\operatorname{Tr} \delta \mathbf{R}\rangle=N \delta L / l, \quad\langle\operatorname{Tr} \delta \mathbf{T}\rangle=N+N(\gamma-1) \delta L / l,
$$

where we have defined $\gamma=\bar{\sigma} l$ The mean free path $l$ in Eq (38) is related to the mean free path $l_{\text {tr }}$ of radiative transfer theory by [18]

$$
\begin{array}{ll}
l=(4 / 3) l_{\mathrm{t}} & \text { for a } 3 \mathrm{D} \text { waveguide } \\
l=(\pi / 2) l_{\mathrm{t} 1} & \text { for a } 2 \mathrm{D} \text { waveguide }
\end{array}
$$

This completes the specification of the statistical ensemble for $\delta S$

We need the increments $\Delta \mathscr{R}_{n}, \Delta \mathscr{T}_{n}$ of reflection and tiansmission eigenvalues to first order in $\delta L / l$,

$$
\begin{gathered}
\Delta \mathscr{R}_{n}=\Delta R_{n n}^{(1)}+\Delta R_{n n}^{(2)}+\sum_{m \neq n} \frac{\Delta R_{m m}^{(1)} \Delta R_{m n}^{(1)}}{\mathscr{R}_{n}-\mathscr{R}_{m}}, \\
\Delta \mathscr{T}_{n}=\Delta T_{n n}^{(1)}+\Delta T_{n n}^{(2)}+\sum_{m \neq n} \frac{\Delta T_{m m}^{(1)} \Delta T_{m n}^{(1)}}{\mathscr{T}_{n}-\mathscr{T}_{m}}
\end{gathered}
$$

The matrices of perturbation $\Delta R^{(1)}, \Delta R^{(2)}, \Delta T^{(1)}, \Delta T^{(2)}$ aie expressed through unitary matrices $Q=V^{\dagger} V^{\prime}, \tilde{U}=Z^{\prime} U_{0}, \tilde{W}=W_{0} V^{\prime}$ and diagonal matrices T, R, $\delta \mathbf{T}, \delta \mathbf{R}$,

$$
\begin{aligned}
\Delta R^{(1)}= & {[\sqrt{\mathbf{R}} \tilde{U} \sqrt{\delta \mathbf{R}} \tilde{W}(1-\mathbf{R})+\mathrm{Hc}] } \\
\Delta R^{(2)}= & -\sqrt{\mathbf{R}} \tilde{U}(1-\delta \mathbf{T}) \tilde{U}^{\dagger} \sqrt{\mathbf{R}}+\tilde{W}^{\dagger} \delta \mathbf{R} \tilde{W}+\sqrt{\mathbf{R}} \tilde{U} \sqrt{\delta \mathbf{R}} \tilde{W} \mathbf{R} \tilde{W}^{\dagger} \sqrt{\delta \mathbf{R}} \tilde{U}^{\dagger} \sqrt{\mathbf{R}} \\
& -\left[\frac{1}{2} \tilde{W}^{\dagger}(1-\delta \mathbf{T}) \tilde{W} \mathbf{R}+\sqrt{\mathbf{R}} \tilde{U} \sqrt{\delta \mathbf{R}} \tilde{W} \sqrt{\mathbf{R}} \tilde{U} \sqrt{\delta \mathbf{R}} \tilde{W}(1-\mathbf{R})+\mathrm{Hc}\right] \\
\Delta T^{(1)}= & -\left[Q \sqrt{\mathbf{R}} \tilde{U} \sqrt{\delta \mathbf{R}} \tilde{W} Q^{\dagger} \mathbf{T}+\mathbf{H} \mathrm{c}\right] \\
\Delta T^{(2)}= & Q \sqrt{\mathbf{R}} \tilde{U} \sqrt{\delta \mathbf{R}} \tilde{W} Q^{\dagger} \mathbf{T} Q \tilde{W}^{\dagger} \sqrt{\delta \mathbf{R}} \tilde{U}{ }^{\dagger} \sqrt{\mathbf{R}} Q^{\dagger} \\
& -\left[\frac{1}{2} Q \tilde{W}^{\dagger}(1-\delta \mathbf{T}) \tilde{W} Q^{\dagger} \mathbf{T}-Q \sqrt{\mathbf{R}} \tilde{U} \sqrt{\delta \mathbf{R}} \tilde{W} \sqrt{\mathbf{R}} \tilde{U} \sqrt{\delta \mathbf{R}} \tilde{W} Q^{\dagger} \mathbf{T}+\mathrm{Hc}\right]
\end{aligned}
$$


(The abbieviation $\mathrm{Hc}$ stands for Hermitian conjugate) The moments (3 2) are computed by first avelaging over the unitary matrices $U_{0}, W_{0}$ and then averaging over $\delta \mathbf{R}$ and $\delta \mathbf{T}$ using Eq (38) Averages ove unitaty matıces follow fiom

$$
\begin{aligned}
& \left\langle U_{n l} U_{m l}^{*}\right\rangle=\frac{1}{N} \delta_{m n} \delta_{k l}, \\
& \left\langle U_{n k} U_{m k} U_{p l}^{*} U_{q l}^{*}\right\rangle=\frac{1}{N(N+1)} \delta_{k l}\left(\delta_{n p} \delta_{m q}+\delta_{m q} \delta_{m p}\right)
\end{aligned}
$$

Without time-1eversal symmetry aveiages over $U_{0}$ and $W_{0}$ are independent With timeteversal symmetry we have $W_{0}=U_{0}^{\mathrm{T}}$ so that only a single average remains The results ate as follows

With time-reversal symmetry

$$
\begin{aligned}
& (l / \delta L)\left\langle\delta \mathscr{R}_{n}\right\rangle=1+2(\gamma-1) \mathscr{R}_{n}+\frac{\mathscr{R}_{n}}{N+1}\left(\mathscr{R}_{n}+\sum_{m} \mathscr{R}_{m}\right) \\
& +\frac{1}{N+1} \sum_{m \neq n} \frac{\mathscr{R}_{n}\left(1-\mathscr{R}_{m}\right)^{2}+\mathscr{R}_{m}\left(1-\mathscr{R}_{n}\right)^{2}}{\mathscr{R}_{n}-\mathscr{R}_{m}}, \\
& (l / \delta L)\left\langle\delta \mathscr{R}_{n} \delta \mathscr{R}_{m}\right\rangle=\frac{4 \delta_{n m}}{N+1} \mathscr{R}_{n}\left(1-\mathscr{R}_{n}\right)^{2}, \\
& (l / \delta L)\left\langle\delta \mathscr{T}_{n}\right\rangle=\mathscr{T}_{n}(\gamma-1)+\frac{\mathscr{T}_{n}}{N+1} \\
& \times\left(A_{n n}+\sum_{m \neq n} \frac{\mathscr{T}_{m} A_{m n}+\mathscr{T}_{n} A_{m m}}{\mathscr{T}_{n}-\mathscr{T}_{m}}+F_{m}+\sum_{m \neq n} F_{n m} \frac{\mathscr{T}_{n}+\mathscr{T}_{m}}{\mathscr{T}_{n}-\mathscr{T}_{m}}\right), \\
& (l / \delta L)\left\langle\delta \mathscr{T}_{n} \delta \mathscr{T}_{m}\right\rangle=\frac{2}{N+1}\left(\delta_{n m} \mathscr{T}_{n}^{2} A_{m}+\mathscr{T}_{n} \mathscr{T}_{m} F_{n m}\right), \\
& (l / \delta L)\left\langle\delta \mathscr{T}_{n} \delta \mathscr{R}_{m}\right\rangle=-\frac{4}{N+1} \mathscr{T}_{n} \mathscr{R}_{m}\left(1-\mathscr{R}_{m}\right)\left|Q_{n m}\right|^{2}
\end{aligned}
$$

Without tme-reversal symmetry

$$
\begin{aligned}
(l / \delta L)\left\langle\partial \mathscr{R}_{n}\right\rangle=1 & +2(\gamma-1) \mathscr{R}_{n}+\frac{\mathscr{R}_{n}}{N} \sum_{m} \mathscr{R}_{m} \\
& +\frac{1}{N} \sum_{m \neq n} \frac{\mathscr{R}_{n}\left(1-\mathscr{R}_{m}\right)^{2}+\mathscr{R}_{m}\left(1-\mathscr{R}_{n}\right)^{2}}{\mathscr{R}_{n}-\mathscr{R}_{m}} \\
(l / \delta L)\left\langle\delta \mathscr{R}_{n} \delta \mathscr{R}_{m}\right\rangle= & \frac{2 \delta_{n m}}{N} \mathscr{R}_{n}\left(1-\mathscr{R}_{n}\right)^{2}
\end{aligned}
$$




$$
\begin{aligned}
& (l / \delta L)\left\langle\delta \mathscr{T}_{n}\right\rangle=\mathscr{T}_{n}(\gamma-1)+\frac{\mathscr{T}_{n}}{N}\left(A_{n n}+\sum_{m \neq n} \frac{\mathscr{T}_{m} A_{m}+\mathscr{T}_{n} A_{m m}}{\mathscr{T}_{n}-\mathscr{T}_{m}}\right), \\
& (l / \delta L)\left\langle\delta \mathscr{T}_{n} \delta \mathscr{T}_{m}\right\rangle=\frac{2 \delta_{n m}}{N} \mathscr{T}_{n}^{2} A_{m n}, \\
& (l / \delta L)\left\langle\delta \mathscr{T}_{n} \delta \mathscr{R}_{m}\right\rangle=-\frac{2}{N} \mathscr{T}_{n} \mathscr{R}_{m}\left(1-\mathscr{R}_{m}\right)\left|Q_{n m}\right|^{2}
\end{aligned}
$$

We have abbreviated $A_{n m}=\left(Q \mathbf{R} Q^{\dagger}\right)_{n m}$ and $F_{n m}=\left|\left(Q \sqrt{\mathbf{R}} Q^{\mathrm{T}}\right)_{m n}\right|^{2}$

The moments of $\delta \mathscr{R}_{n}$ contain only the set of reflection eigenvalues $\left\{\mathscr{R}_{n}\right\}$, so that from $\mathrm{Eq} \mathrm{(3} \mathrm{3)} \mathrm{we} \mathrm{can} \mathrm{1mmediately} \mathrm{write} \mathrm{down} \mathrm{a} \mathrm{Fokker-Planck} \mathrm{equation} \mathrm{for} \mathrm{the}$ distribution of the $\mathscr{R}_{n}$ 's In terms of variables $\mu_{n}=1 /\left(\mathscr{R}_{n}-1\right) \in(-\infty,-1) \cup(0, \infty)$ it reads [16]

$$
\begin{aligned}
l \frac{\partial}{\partial L} P\left(\left\{\mu_{n}\right\}, L\right)= & \frac{2}{\beta N+2-\beta} \sum_{n=1}^{N} \frac{\partial}{\partial \mu_{n}} \mu_{n}\left(1+\mu_{n}\right) \\
& \times\left[\frac{\partial P}{\partial \mu_{n}}+\beta P \sum_{m \neq n} \frac{1}{\mu_{m}-\mu_{n}}+\gamma(\beta N+2-\beta) P\right],
\end{aligned}
$$

where the symmetry index $\beta=1(2)$ corresponds to the case of unbroken (broken) time-1eversal symmetry The evolution of the reflection eigenvalues is independent of the transmission eigenvalues - but not vice versa The evolution of the $\mathscr{T}_{n}$ 's depends on the $\mathscr{R}_{n}$ 's, and in addition on the slow valuables contained in the unitaiy matix $Q$ To obtain a closed Fokke-Planck equation we also need to compute increments and moments of $Q$ The resulting expressions are lengthy and w1ll not be wiltten down here

In the single-mode case $(N=1)$ this complication does not arise, because $Q=e^{i \phi}$ drops out of the scalars $A$ and $F$ The single transmission and reflection eigenvalues $\mathscr{T}, \mathscr{R}$ coincide with the transmittance and reflectance $T, R$ defined by Eq (22) The resultıng Fokker-Planck equation is [9]

$$
\begin{aligned}
l \frac{\partial P}{\partial L}= & -\frac{\partial}{\partial R}\left[(1-R)^{2}+2 \gamma R\right] P+\frac{\partial^{2}}{\partial R^{2}} R(1-R)^{2} P \\
& -\frac{\partial}{\partial T} T(\gamma-1+R) P+\frac{\partial^{2}}{\partial T^{2}} T^{2} R P-2 \frac{\partial^{2}}{\partial T \partial R} T R(1-R) P
\end{aligned}
$$

In the case of absorption $(\gamma<0)$ Eq $(316)$ is equivalent to the moments equations [5]

\section{Localization length}

The limit $L \rightarrow \infty$ of the distribution of the reflection eigenvalues follows ditectly from $\mathrm{Eq}$ (3 15), by equating the left-hand-side to zero The 1esulting distribution $P_{\infty}$ 
is that of the Laguene ensemble of 1andom mat11x theory [16],

$$
P\left(\left\{\mu_{l}\right\}\right) \propto \prod_{i<1}\left|\mu_{l}-\mu_{i}\right|^{\beta} \prod_{k} \exp \left[-\gamma(\beta N+2-\beta) \mu_{k}\right]
$$

The distribution looks the same for both signs of $\gamma$, but the suppott (and the normalization constant) is different $\mu_{n}>0$ for $\gamma>0$, and $\mu_{n}<-1$ for $\gamma<0$ To determine the localization length we need the distribution of the transmission eigenvalues in the laige- $L$ limit We consider the cases $N=1$ and $N \gg 1$

\section{Sinqle-mode wavegute}

We compute the disti1bution $P(T, L)$ of the transmission probability thiough a singlemode waveguide in the limit $L \rightarrow \infty$ In the case of absoiption $(\gamma<0)$ this calculation was done by Rammal and Doucot [4], and by Fre1lıkher et al [5] We generalize the11 results to the case of amplification $(\gamma>0)$ The two cases are essentially different because, while the mean value of $R$ is finte in the case of absorption,

$$
\langle R\rangle_{\infty}=1-2 \gamma e^{2 \gamma} \operatorname{E} 1(2 \gamma) \text { for } \gamma<0,
$$

it diverges in the case of amplification The mean value of $\ln R$ is finite in both cases,

$$
\langle\ln R\rangle_{\infty}= \begin{cases}C+\ln 2 \gamma-e^{2 \gamma} \operatorname{E}(-2 \gamma) & \text { for } \gamma>0, \\ -C-\ln (-2 \gamma)+e^{2 \gamma} \operatorname{Er}(2 \gamma) & \text { for } \gamma<0\end{cases}
$$

Here $C$ is Euler's constant and $\mathrm{E} 1(x)=\int_{\infty}^{x} d t e^{t} / t$ is the exponentral integral The telation

$$
\langle\ln R(\gamma)\rangle_{\infty}=-\langle\ln R(-\gamma)\rangle_{\infty}
$$

holds, in accordance with the duality ielation (2 5)

We now show that the asymptotic $L \rightarrow \infty$ distribution of $T$ is $\log$-normal, with mean and variance of $\ln T$ given by

$$
\begin{gathered}
\langle\ln T\rangle=-(1+|\gamma|) L / l+2 c(\gamma)+\mathcal{O}(l / L), \\
c(\gamma)= \begin{cases}0 & \text { for } \gamma<0, \\
C+\ln 2 \gamma-e^{2 \gamma} \operatorname{El}(-2 \gamma) & \text { for } \gamma>0,\end{cases} \\
\text { var } \ln T=\left[2+4|\gamma| e^{2|/|} \operatorname{El}(-2|\gamma|)\right] L / l+\mathcal{O}(1)
\end{gathered}
$$

The constant $c(\gamma) \approx-2 \gamma \ln \gamma$ if $0<\gamma \ll 1$ Note that val $\ln T \ll\langle\ln T\rangle^{2}$ for $L / l \gg 1$ The localization length $\xi=l(1+|\gamma|)^{1}$ is independent of the sign of $\gamma$, in accordance with the duality relation $\left(\begin{array}{ll}1 & 2\end{array}\right)$

These results are easy to establish for the case of absoiption, when Eq ( 316$)$ implies the evolution equations [4,5]

$$
l \frac{\partial}{\partial L}\langle\ln T\rangle=-1+\gamma, \quad l \frac{\partial}{\partial L} \text { val } \ln T=2\langle R\rangle, \quad \text { for } \gamma<0
$$


Making use of the initial condition $T \rightarrow 1$ for $L \rightarrow 0$ and the asymptotic value (42) of $\langle R\rangle$, one readily obtains Eqs (45) and (46) for $\gamma<0$

In the case of amplification, the evolution equations (47) hold only for lengths $L$ smaller than $L_{c} \simeq l c(\gamma) /|\gamma|$ For $L \lesssim L_{c}$ stimulated emission enhances tiansmission through the waveguide On larger length scales stimulated emission reduces transmission Technically, the evolution equations (47) break down for $L \rightarrow \infty$ because the integration by parts of the Fokker-Planck equation produces a non-zero boundary term if $L>L_{c}$ To extend Eqs (45) and (46) to the case $\gamma>0$ we use the duality relation (24) It implies that for $N=1$ the distribution of the 1 atio $T / R$ is an even function of $\gamma \mathrm{Eq}$ (45) for $\gamma>0$ follows directly from the equality

$$
\langle\ln T(\gamma) / R(\gamma)\rangle=\langle\ln T(-\gamma) / R(-\gamma)\rangle,
$$

which holds for all $L$, plus Eq (44), which holds for $L \rightarrow \infty$ The constant $c(\gamma)$ for $\gamma>0$ equals $\langle\ln R(\gamma)\rangle_{\infty}$ and is substituted from $\mathrm{Eq}$ (43) The duality of $T(\gamma) / R(\gamma)$ also implies Eq (46) for the variance, provided the covariance $\langle\langle\ln T \ln R\rangle\rangle=\langle\ln T \ln R\rangle-$ $\langle\ln T\rangle\langle\ln R\rangle$ remains finite as $L \rightarrow \infty$ We have checked this ditectly fiom the Fokker Planck equation (3 16), and found the finite large- $L$ limit

$$
\begin{aligned}
\langle\langle\ln T \ln R\rangle\rangle_{\infty}= & -2 e^{2 \gamma} \operatorname{E} 1(-2 \gamma) c(\gamma)-c(\gamma)^{2} \\
& -2 \gamma \int_{0}^{\infty} d \mu e^{-2 \gamma \mu}\left[\ln ^{2}(1+\mu)-\ln ^{2} \mu\right] \text { for } \gamma>0
\end{aligned}
$$

\section{Mult - -mode waveguide}

We next consider a waveguide with $N \gg 1$ modes We compute the localization length $\xi=-\lim _{L \rightarrow \infty} L^{-1}\langle\ln T\rangle$ in the case of absorption, and include the case of amplification invoking duality For absoiption the average reflectance $\langle R\rangle=N^{-1}\left\langle\sum_{k}\left(1+1 / \mu_{k}\right)\right\rangle$ remains finite as $L \rightarrow \infty$ The large- $L$ limit $\langle R\rangle_{\infty}$ follows from the distribution (4 1), using known formulas for the eigenvalue density in the Laguerre ensemble [22] For $|\gamma| N^{2} \gg 1$ the result is

$$
\langle R\rangle_{\infty}=1+|\gamma|-\sqrt{|\gamma|(2+|\gamma|)}+\mathcal{O}(1 / N), \quad \gamma<0
$$

The evolution of transmission eigenvalues is governed by the Fokke1-Planck equation (3 3), with coefficients given by (32), (3 13) and (3 14) Each $\mathscr{T}_{n}$ has its own localization length $\xi_{n}=-\lim _{L \rightarrow \infty} L^{-1} \ln \mathscr{T}_{n}$ We order the $\xi_{n}$ 's from la1ge to small, $\xi_{1}>\xi_{2}>>\xi_{\Lambda}$ This implies that for $L \rightarrow \infty$ the separation of the $\mathscr{T}_{n}$ 's becomes exponentially large, $\mathscr{T}_{1} \gg \mathscr{T}_{2} \gg \quad \gg \mathscr{T}_{N}$ Hence we may approximate

$$
\begin{aligned}
& \frac{\mathscr{T}_{n}+\mathscr{T}_{m}}{\mathscr{T}_{n}-\mathscr{T}_{m}} \approx \begin{cases}-1 & \text { for } n>m, \\
1 & \text { for } n<m,\end{cases} \\
& \frac{\mathscr{T}_{n} A_{m n}+\mathscr{T}_{m} A_{n n}}{\mathscr{T}_{n}-\mathscr{T}_{m}} \approx \begin{cases}-A_{n n} & \text { for } n>m, \\
A_{m m} & \text { for } n<m\end{cases}
\end{aligned}
$$


The Fokke-Plank equation (3 3) simplifies considerably and leads to the following equation for the laigest transmission eigenvalue

$$
l \frac{\partial}{\partial L}\left\langle\ln \mathscr{T}_{1}\right\rangle= \begin{cases}-1-|\gamma|+\langle R\rangle-\frac{1}{N+1}\left\langle A_{11}+F_{11}\right\rangle & \text { for } \beta=1, \\ -1-|\gamma|+\langle R\rangle-\frac{1}{N}\left\langle A_{11}\right\rangle & \text { for } \beta=2\end{cases}
$$

For $|\gamma| N^{2} \gg 1$ we may substitute $\mathrm{Eq}(410)$ for $\langle R\rangle$ and omit the teims with $\left\langle A_{11}\right\rangle$ and $\left\langle F_{11}\right\rangle$ The resulting localization length is given by

$$
l / \xi=\sqrt{|\gamma|(2+|\gamma|)}+\mathscr{O}(1 / N)
$$

Because of duality, Eq (4 13) holds regardless of the sign of $\gamma$ It agrees with radiative transfer theory for $\gamma<0$, but not for $\gamma>0$ Indeed, the exponentral decay of the transmitted intensity in the case of amplification is an interference effect, which is not contained in the theory of radiative transfer

Eq (4 13) 1s asymptotically exact for $|\gamma| \gg 1 / N^{2}$ For smallet $|\gamma|$ we cannot compute $\xi$ 1igorously because the distibution of the matuces $\mathbf{A}$ and $\mathbf{F}$ is not known An interpolative formula for all $\gamma$ can be obtained by substituting for $\left\langle A_{11}\right\rangle$ and $\left\langle F_{11}\right\rangle$ in Eq (4 12) the11 $L \rightarrow \infty$ limits when $\gamma=0$, which ate $\left\langle A_{11}\right\rangle=\left\langle F_{11}\right\rangle=1$ In this way, we arrive at the localization length

$$
\xi=l\left[\frac{2}{\beta N+2-\beta}+\sqrt{|\gamma|(2+|\gamma|)}\right]^{-1},
$$

which interpolates between the known $[13,14,23]$ value of $\xi$ for $\gamma=0$ and Eq (4 13) for $|\gamma| \gg 1 / N^{2}$

The localization length $\xi$ is the laigest of the ergenvalue-dependent localization lengths $\xi_{n}$ What about the other $\xi_{n}$ 's? For $\gamma=0$ it 1s known $[14,15,23]$ that the inverse localization lengths are equally spaced, and satisfy the sum rule $\sum_{n} 1 / \xi_{n}=N / l$ We have not succeeded in deriving the spacings for $\gamma \neq 0$, but we have been able to detive the sum iule fiom the Fokker Planck equation (by computing the $L$-dependence of $\left.\left\langle\sum_{n} \ln \mathscr{T}_{n}\right\rangle\right)$ The 1esult is exact and reads

$$
l \sum_{n-1}^{N} \xi_{n}^{-1}=(1+|\gamma|) N
$$

\section{Numerical results}

To test the analytical predictions on a model system, we have numerically solved a discietized veision of the Helmholtz equation,

$$
\left[\nabla^{2}+k^{2} \varepsilon(\boldsymbol{r})\right] E(\boldsymbol{r})=0
$$

on a two-dimensional square lattice (lattice constant $d$, length $L$, width $W$ ) The real patt $\varepsilon^{\prime}$ of the dielectic constant was chosen iandomly fiom site to site with a uniform 


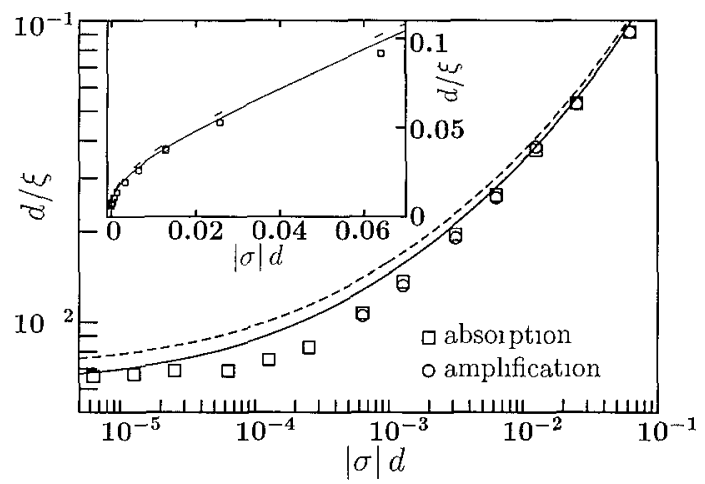

Fig 1 Localization length $\xi=-\lim _{L \rightarrow \infty} L^{1}{ }^{1}\langle\ln T\rangle$ of a disordesed waveguide $(N=10)$ versus the modal avcrage $\bar{\sigma}$ of the inverse absorption or amplification length Data ponts are numencal solutions of the discietized (lattice constant $d$ ) two dimensional Helmholte equation for the case of absotption (squaies) and amplrfication (circles) The curves are the analytical prediction (414) for the case $\beta=1$ (unbioken time-reversal symmetry) for $l=296 d$ [sold curve, determuncd fiom Eq (5 3)] and for $l=261 d$ [dashed curve, determined from Eq (54)] The inset shows the same data on a lineal, rather than logat thmic, scale

distribution between $1 \pm \delta \varepsilon$ The imaginary part $\varepsilon^{\prime \prime}$ was the same at all sites The scattering matrix was computed using the recuisive Green function technique [24]

The parameter $\bar{\sigma}$ is obtained from the analytical solution of the discretized Helmholtz equation in the absence of disorde1 $(\delta \varepsilon=0)$ The complex longitudinal wavenumber $k_{n}$ of transverse mode $n$ then satisfies the dispersion relation

$$
\cos \left(k_{n} d\right)+\cos (n \pi d / W)=2-\frac{1}{2}(k d)^{2}\left(1+1 \varepsilon^{\prime \prime}\right),
$$

which determines $\bar{\sigma}$ according to $\bar{\sigma}=-2 N^{-1} \operatorname{Im} \sum_{n} k_{n}$ Simulations with $\varepsilon^{\prime \prime}=0$ weie used to obtain $l$, eithe1 from the large- $L$ relation [14]

$$
-\lim _{l \rightarrow \infty} L^{-1}\langle\ln T\rangle=\left[\frac{1}{2}(N+1) l\right]{ }^{1},
$$

or from the large- $N$ relation [25]

$$
\lim _{N \rightarrow \infty}\langle T\rangle=(1+L / l)^{-1}
$$

The parameters chosen were $W=25 d, k=122 d^{-1}$, coiresponding to $N=10, l=296 d$ fiom $\mathrm{Eq}(53)$ and $l=261 d$ from $\mathrm{Eq}$ (54) The localization length was computed as a function of $\sigma$ from the $L$-dependence of $\ln T$ up to $40 l$, averaged over 150 realizations of the disorder Results are shown in F1g 1 The localization length is the same for absorption and amplification, within the numerical accuracy Comparison with the analytical result $(414)$ for $\beta=1$ s plotted for the two values of the mean fiee path The agreement is quite reasonable, given the approximate nature of Eq (4 14) in the regime $|\gamma| N^{2} \simeq 1$ (corresponding to $|\bar{\sigma}| d \simeq 10^{-4}$ )

In conclusion, we have shown how absorption or amplification can be incorporated into the Dorokhov-Mello-Pereyra-Kumai equation for transmission through a mult1-mode waveguide The technical difficulty of the mult1-mode case is that the 
Fokke1-Planck equation for the transmission eigenvalues $\mathscr{T}_{n}$ depends not just on the transmission and reflection eigenvalues $\mathscr{T}_{n}, \mathscr{R}_{n}$, but also on the eigenvectors of the matices $t t^{\prime}$ and $r r^{\dagger}$ We could compute the localization length in the two regimes, $|\gamma| \gg 1 / N^{2}$ and $|\gamma| \ll 1 / N^{2}$, and have given an interpolation formula for the inteimediate regime An exact solution for all $\gamma$ remains an unsolved problem

\section{Acknowledgements}

We acknowledge useful discussions with PW Brouwer and KM Fiahm This research was suppoited in part by the "Nederlandse organisatie voor Wetenschappelijk Ondeizoek" (NWO) and by the "Stichtıng voor Fundamenteel Ondeizoek der Materie" (FOM)

\section{References}

[1] P E1dos and R C Heindon, Adv Phys 31 (1982) 65

[2] J B Pendiy, Adv Phys 43 (1994) 461

[3] ME Gertsenshteın and VB Vas1'ev, Teor Vcioyatn Pıimen 4 (1959) 4245 (1960) 3(E) [Theo1 Probab Appl 4 (1959) 391, 5 (1960) 340(E)]

[4] R Rammal and B Doucot, J Phys (Ftance) 48 (1987) 509

[5] V Fteilıkheı, M Pustılnik and I Yutkevich, Phys Rev Lett 73 (1994) 810

[6] P Pladhan and N Kuma1, Phys Rev B 50 (1994) 9644

[7] A K Gupta and A M Jayannaval, Phys Rev B 52 (1995) 4156

[8] Z-Q Zhang, Phys Rev B 52 (1995) 7960

[9] J C J Paasschens, T Sh Misırpashaev and C W J Beenakke1, Phys Rev B 54 (1996) 11887

[10] V Fieılıker, M Pustılnık, and I Yurkevich, prepıint (cond-mat/9605090)

[11] A K Sen, Mod Phys Lett B 10 (1996) 125

[12] D J Thouless, Phys Rev Lett 39 (1977) 1167

[13] K B Efetov and A I Lakkn, Zh Eksp Teov Fiz 85 (1983) 764 [Sov Phys JETP 58 (1983) 444]

[14] ON Dorokhov, Pisma Zh Eksp Teor Fiz 36 (1982) 259 [JETP Lett 36 (1982) 318], Zh Eksp Teor Fiz 85 (1983) 1040 [Sov Phys JETP 58 (1983) 606]

[15] P A Mello, P Peieyıa, and N Kumar Ann Phys (N Y) 181 (1988) 290

[16] C W I Bcenakket, J C J Paasschens and P W Brouwer, Phys Rev Lett 76 (1996) 1368

[17] N A Bruce and J T Chalke1, J Phys A 29 (1996) 3761

[18] Fot a teview of the theory of the DMPK equation, see CW J Beenakke1, Rev Mod Phys (to be published)

[19] G L J A Rikken and B A van Tiggelen, Naturc 381 (1996) 54

[20] N G van Kampen, Stochastic Processcs 1n Physics and Chemistry (North Holland, Amsteidam, 1981)

[21] P A Mello and S Tomsovic, Phys Rev B 46 (1992) 15963

[22] T Nagao and K Slevin, J Math Phys 34 (1993) 2075, 34 (1993) 2317

[23] A D Stonc, PA Mcllo, KA Muttalib and J-L Pichaid, in Mesoscopic Phenomena in Solids, eds B L Al tshulet, PA Lce and R A Wcbb (North-Holland, Amsterdam, 1991)

[24] H U Barange, D P DiVincenzo, R A Jalabeit and A D Stone, Phys Rev B 44 (1991) 10637

[25] PA Mello and A D Stone, Phys Rev B 44 (1991) 3559 